\title{
Post Hoc Evidence for an Additive Effect of Memantine and Donepezil: Consistent Findings from DOMINO-AD Study and Memantine Clinical Trial Program
}

\author{
S. Hendrix ${ }^{1}$, N. Ellison ${ }^{1}$ S. Stanworth ${ }^{1}$, V. Otcheretko ${ }^{2}$, P.N. Tariot ${ }^{3}$

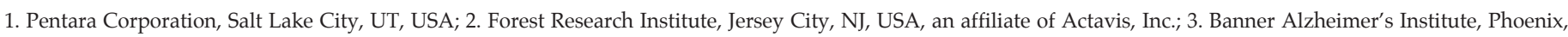 \\ AZ, USA \\ Corresponding Author: Suzanne Hendrix, Pentara Corporation, Salt Lake City, UT, USA, Telephone: ++1-801-898-724, Fax: ++1-801-486-7467, shendrix@pentaracorp.com
}

Published online May 12, 2015, http:/ / dx.doi.org/10.14283/jpad.2015.66

\begin{abstract}
BACKGROUND: Several randomized trials have demonstrated superiority of memantine-cholinesterase inhibitor combination therapy in patients with moderate to severe Alzheimer's disease, yet a recent publication reported no additional benefit of add-on memantine therapy compared to donepezil alone.

OBJECTIVES: In this post hoc analysis, we sought to re-evaluate the results from the DOMINO study using common statistical tools and to apply the statistical models used in the DOMINO study to a pooled data set of 24- to 28-week randomized trials of memantine in patients with moderate to severe $\mathrm{AD}$ in order to explore the robustness of the primary findings from the DOMINO study.
\end{abstract}

DESIGN: DOMINO study: Randomized, double-blind, placebo-controlled trial (Current Controlled Trial number, ISRCTN49545035); Memantine Clinical Trial Program: Pooled analysis from four randomized, double-blind, placebocontrolled trials.

SETTING: DOMINO study: United Kingdom; Memantine Clinical Trial Program: Multinational.

PARTICIPANTS: DOMINO study: 295 participants enrolled during the period of February 2008 to March 2010; Memantine Clinical Trial Program: 1417 participants enrolled between August 1998 and January 2008.

MEASUREMENTS: In the DOMINO study, the co-primary outcome measures were scores on the Standardized MiniMental State Examination and the Bristol Activities of Daily Living Scale; Neuropsychiatric Inventory was a secondary measure. In the Memantine Clinical Trial Program, outcome measures included the Severe Impairment Battery, the 19-item Alzheimer's Disease Cooperative Study - Activities of Daily Living scale, Neuropsychiatric Inventory, and a 4-Domain Composite Index (Z-score; a post hoc assessment).

RESULTS: Both the pooled analysis of the Memantine Clinical Trial Program and the re-assessment of the DOMINO study with common statistical tools showed that adding memantine to donepezil therapy is associated with benefits across multiple clinical domains.

CONCLUSIONS: The current analyses suggest that the results of the DOMINO study do not contradict previous studies which investigated the combined effects of memantine-cholinesterase inhibitor treatment.

Key words: Memantine, donepezil, combination therapy, treatment strategy.

\section{Introduction}

7 reatment guidelines and health policy decisions critically depend on the outcomes of clinical trials and the conclusions reported by the investigators. However, sources of uncertainty and bias - eg, chance, sample size, enrollment criteria, study design, data analysis plan, choice of statistical tools (1) remain inherent to any clinical study.

Therefore, it is common for seemingly similar studies, or a formal reanalysis of trial outcomes, to result in different conclusions or conclusions that are contrary to previous results (2). For example, several lines of evidence, including 6-month randomized clinical trials (3-6), post hoc analyses $(7,8)$, and long-term (1-14 year) observational cohort effectiveness studies (9-11), suggest that memantine-cholinesterase inhibitor (ChEI) combination therapy in patients with moderate to severe $\mathrm{AD}$ is superior to monotherapy with either drug or drug class. In contrast, the authors of a randomized, 52-week Donepezil and Memantine in Moderate to Severe Alzheimer's Disease (DOMINO) study (12) concluded, based on a subgroup analysis, that the addition of memantine to ongoing donepezil treatment was not superior to donepezil or memantine monotherapy. However, due to a variety of methodological limitations, including small sample size, the failure to demonstrate a «statistically significant» effect of addition of memantine does not mean or even imply that there is no effect (see Discussion). The current treatment paradigm for AD consists of monotherapy with a ChEI (eg, donepezil) in the earlier stages (13) and the addition of memantine in the moderate or severe stages (14-16). Reconciling the apparently dissimilar conclusions of the DOMINO publication and the previous clinical trial results is crucial for determining the best standard of care for patients with moderate to severe $\mathrm{AD}$, and is the goal of this paper.

The purpose of the current analysis was twofold: (1) Reassess the published DOMINO results using common statistical tools, and (2) apply the statistical models from the DOMINO study to a pooled data set of 24- to 28-week 
randomized trials of memantine in moderate to severe $\mathrm{AD}(3-6)$ in order to examine the statistical support for the DOMINO conclusions.

\section{Methods}

\section{Trial Characteristics and Treatment Groups}

\section{DOMINO Study (12)}

The DOMINO study was a one-year, multicenter, double-blind, placebo-controlled, clinical trial with a two-by-two factorial design. The participants were community-dwelling individuals with moderate to severe probable AD ( $\mathrm{N}=295)$ (Standardized Mini-Mental State Examination [SMMSE] score range: 5-13) who had been receiving donepezil continuously for $\geq 3$ months with a daily dose of $10 \mathrm{mg}$ for $\geq 6$ weeks and whose prescribing physicians were considering change of treatment (stopping donepezil or introducing memantine). A randomized minimization procedure and stratification by donepezil treatment duration (3-6 months or $>6$ months), age ( $<60$ years, $60-74$ years, or $>74$ years), and SMMSE score (5-9 or 10-13) were used to assign participants to one of the following treatments: discontinue donepezil $(\mathrm{n}=73)$, discontinue donepezil and start $20 \mathrm{mg}$ memantine $(\mathrm{n}=76)$, continue $10 \mathrm{mg}$ donepezil $(\mathrm{n}=73)$, or continue donepezil $10 \mathrm{mg}$ and start $20 \mathrm{mg}$ memantine $(\mathrm{n}=73)$.

\section{Memantine Clinical Trial Program}

The four pooled trials of the Memantine Clinical Trial Program were 6-month, randomized (1:1), multicenter, parallel-group, double-blind, and placebo-controlled. Outpatients $(\mathrm{N}=1,957)$ met standardized clinical criteria for moderate to severe probable AD (combined MMSE range: 3-16) and were either not receiving background antidementia therapy $(4,5)$ (memantine monotherapy trials) or receiving ChEI therapy continuously for $\geq 6$ months, of which a stable dose for $\geq 3$ months $(3,6)$ (add-on/combination trials). The active treatment was memantine (10 mg bid) (3-5) or memantine extendedrelease $(28 \mathrm{mg}$ qd) (6). The pooled populations ( $\mathrm{n}=1,417$, excluding non-donepezil ChEIs) were divided into four treatment categories created to mimic the DOMINO groups: placebo-treated participants from the two memantine monotherapy trials $(4,5)$ (PBO; $\mathrm{n}=281$ ), memantine-treated participants from the two monotherapy trials $(4,5)(M E M ; n=289)$, placebo-treated participants who were concurrently taking donepezil in the two add-on trials $(3,6)(\mathrm{DON} / \mathrm{PBO} ; \mathrm{n}=418)$, or memantine-treated participants who were concurrently taking donepezil in the two add-on trials $(3,6)(\mathrm{DON} /$ MEM; $n=429)$. Data from participants who were on a ChEI other than donepezil at Baseline were not included.

The treatment groups in the DOMINO study and the pooled dataset of the Memantine Clinical Trial Program are shown in Table 1. It is important to note that all treatment assignments in DOMINO were randomized, whereas in the pooled studies memantine and placebo treatments were randomly assigned but donepezil

Table 1. DOMINO Study and Pooled Sample of the Memantine Clinical Trial Program: Treatment Groups, Assessments, and Statistical Analyses

\begin{tabular}{|l|l|l|}
\hline $\begin{array}{l}\text { Groups } \\
\text { No active treatment }\end{array}$ & DOMINO Study (12) & Memantine Clinical Trial Program (3-6) \\
$\begin{array}{l}\text { Active treatment: MEM } \\
\text { Active treatment: DON }\end{array}$ & Discontinue DON $(\mathrm{n}=73)$ & PBO $(4,5)(\mathrm{n}=281)^{*}$ \\
\hline $\begin{array}{l}\text { Active treatment: DON, MEM } \\
\text { Assessments }\end{array}$ & Discontinue DON start MEM $(\mathrm{n}=76)$ & MEM only $(4,5)(\mathrm{n}=289)^{*}$ \\
\hline $\begin{array}{l}\text { Cognition } \\
\text { Daily Functioning }\end{array}$ & Continue DON start MEM $(\mathrm{n}=73)$ & Continue DON start PBO $(3,6)(\mathrm{n}=418)^{*}$ \\
\hline $\begin{array}{l}\text { Behavior } \\
\text { Composite }\end{array}$ & SMMSE & Continue DON start MEM $(3,6)(\mathrm{n}=429)^{*}$ \\
\hline $\begin{array}{l}\text { Statistical Analysis } \\
\text { Reporting }\end{array}$ & BADLS & \\
\hline & NPI & SIB \\
\hline
\end{tabular}

*Total population, not population with all visit data available; 4D indicates four-dimensional; ADCS-ADL19, the 19-item Alzheimer's Disease Cooperative Study - Activities of Daily Living scale (score range: 0-54); BADLS, Bristol Activities of Daily Living Scale (score range: 0-60); CI, indicates confidence interval; DON, donepezil; MEM, memantine; MMRM, multilevel modeling with repeated measures; NPI, Neuropsychiatric Inventory (score range: 0-144); PBO, placebo; SMMSE, Standardized Mini-Mental State Examination (score range: 0-30); SIB, Severe Impairment Battery (score range: $0-100$ ). 
Table 2. DOMINO and Memantine Clinical Trial Program: Baseline Characteristics

DOMINO Study (Based on Howard et al, 2012) (12)

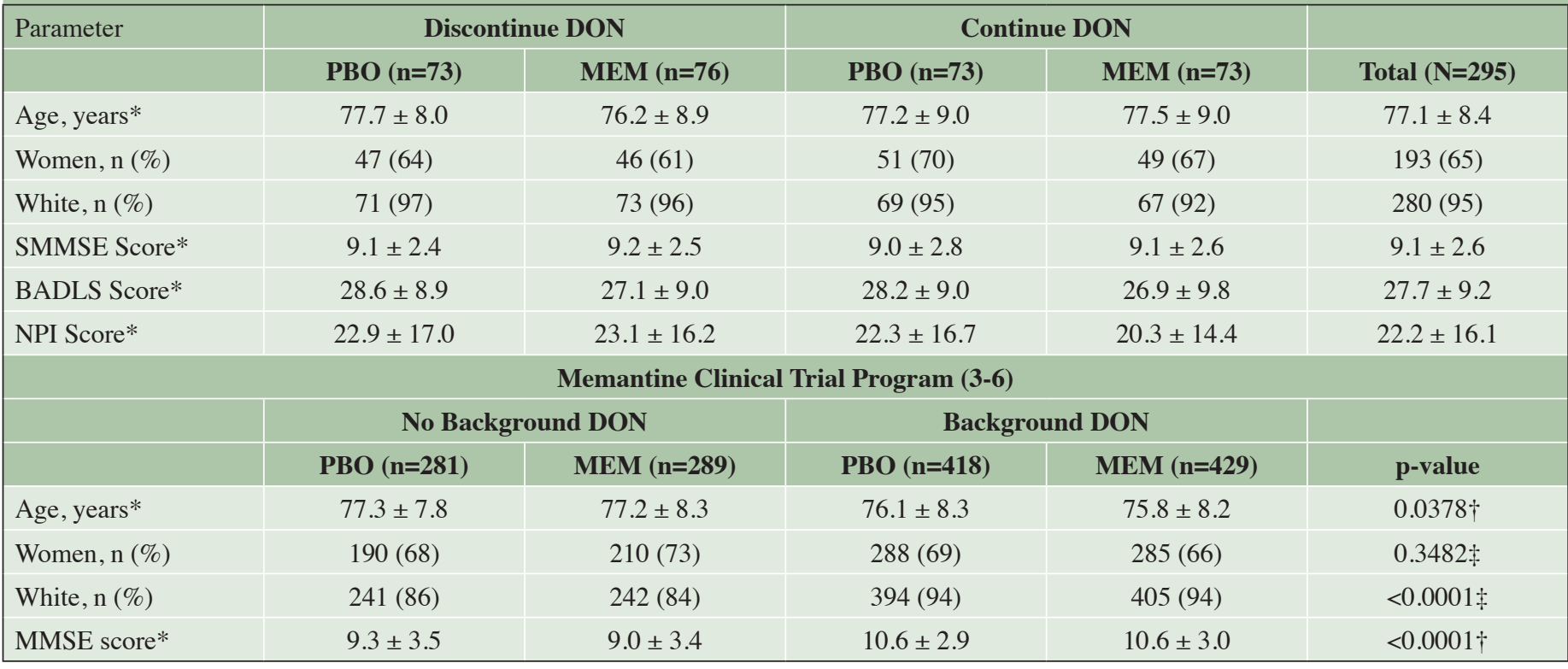

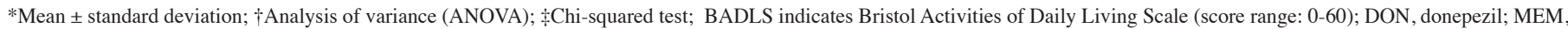

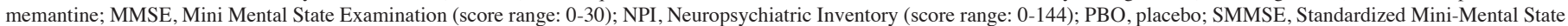
Examination.

pretreatment was not, meaning that observed memantine effects are due to group assignment, but observed donepezil effects could be due to group assignment, selection issues or population differences.

\section{Efficacy Assessments and Outcomes}

In the DOMINO study, the co-primary outcome measures were the Standardized Mini-Mental State Examination (SMMSE) (17) and the Bristol Activities of Daily Living Scale (BADLS) (18). The SMMSE is a 12-item, 30-point scale designed to assess cognitive performance, in which higher scores indicate better cognitive abilities. The BADLS is a 20-item, 60-point scale designed to assess the ability of someone with dementia to carry out daily activities, in which higher scores indicate greater impairment. A secondary outcome measure was the Neuropsychiatric Inventory (NPI) (19), which is a 12-item, 144-point scale designed to assess behavior, with higher scores indicating greater behavioral problems.

In the Memantine Clinical Trial Program, the cognitive outcome measure was the Severe Impairment Battery (SIB) (20), a 40-item, 100-point scale designed to assess cognitive performance in patients with moderate to severe $\mathrm{AD}$, in which lower scores indicate greater impairment. Daily function was assessed using the 19-item Alzheimer's Disease Cooperative Study Activities of Daily Living scale (ADCS-ADL19) (21), a 54-point instrument designed to assess functional abilities in patients with moderate to severe AD, in which lower scores indicate greater impairment. Behavioral symptoms were assessed with the NPI. Lastly, a post hoc 4-Domain (4D) Composite Index (Z-score) was an equally weighted composite measure consisting of the four outcome measures -- SIB, ADCS-ADL19, NPI, and the Clinician's Interview-Based Impression of Change Plus Caregiver Input (CIBIC-Plus), a measure of patient's global clinical status (22) -- based on the distribution of the baseline scores. In order to make the contributing scales uniform in their direction of improvement, the direction of improvement for the NPI and CIBIC-Plus was reversed.

\section{Statistical Analysis}

Results of the DOMINO study were re-assessed using a multilevel modeling regression with repeated measures (MMRM) with effects for memantine, donepezil, and memantine by donepezil interaction, adjusted for baseline scores and for the four stratification factors (center, duration of donepezil treatment before study entry, baseline SMMSE score, and age), with random effects for each visit and a variance component structure that was chosen because an unstructured covariance matrix in the DOMINO study did not converge (12). In addition, confidence intervals (CIs) were converted into p-values (two-sided). Data from the Memantine Clinical Trial Program were analyzed using a similar regression model to that employed in the DOMINO trial by applying the same two covariance structures: an unstructured covariance structure, which did converge for the pooled memantine data, and a variance components structure, which was used for the DOMINO 
results.

The three objectives stated in the DOMINO publication were 1) to assess the main effect of donepezil continuation vs discontinuation, 2) to assess the main effect of memantine addition vs matched placebo, and 3 ) to test whether the addition of memantine to ongoing donepezil treatment would provide additive or synergistic benefits in patients with moderate to severe AD. The distinction between additivity and synergy, according to the publication, was based on homogeneous versus heterogeneous effects for each treatment alone (i.e., monotherapy) versus those of the combined treatments (12). Although the specific statistical tests were not explicitly stated, additivity would be demonstrated if donepezil and memantine treatment effects were homogeneous in the presence and absence of the other treatment, while synergy (either positive or negative) would be consistent with heterogeneous outcomes. Our analysis used the traditional definitions of synergy and additivity, with a significant positive or negative interaction between the two treatments being the criterion for synergy (i.e., heterogeneity), and the absence of a significant interaction in the presence of significant main effects being the criterion for additivity (i.e., homogeneity) (23). In other words, positive or negative synergy would be determined by a combined effect that is larger or smaller, respectively, than the sum of the two individual effects, and that is associated with a significant treatment interaction; a combined effect that is larger or smaller than the sum of the individual effects, but without significant interaction, would indicate additivity.

A comparison of the statistical analyses used for the DOMINO trial and Memantine Clinical Trial Program is presented in Table 1. Baseline demographics and characteristics of the pooled data set were assessed using summary statistics and compared by means of ANOVA (continuous variables) or a chi-squared test (dichotomous variables). No adjustments for multiple comparisons were made (i.e., each variable was considered independently) (Table 2). Center was included as a random effect in the model for the Memantine Clinical Trial Program. Previous time on donepezil was not available for the monotherapy studies included in the Memantine Clinical Trial Program and was therefore not included in the model.

\section{Results}

\section{Patient Demographics and Baseline Characteristics}

In the DOMINO study, a total of 295 participants were enrolled during the period of February 2008 to March 2010. Recruitment was slower than anticipated and the public funder of the study did not allow extending the recruitment period, which resulted in a sample size notably smaller than the one originally planned (800) or the one of 430 that, according to an interim analysis, would have yielded power of $80 \%$ to $96 \%$. The baseline characteristics of the DOMINO study participants and the four study populations from the Memantine Clinical Trial Program are shown in Table 2. In the DOMINO study, the baseline characteristics were similar among treatment groups. In the Memantine Clinical Trial Program, statistically significant differences across groups were primarily due to differences in baseline characteristics between the trials with background donepezil and those without (however, note that each pooled trial was randomized, so the memantine and placebo groups within each donepezil group were comparable).

\section{Efficacy Assessments}

\section{DOMINO Study}

Based on the confidence intervals presented in the primary paper (12), the DOMINO study found a significant overall advantage of continued donepezil versus donepezil discontinuation (with or without memantine addition) across all visits for SMMSE and BADLS, but not for NPI, with p-values based on our reassessment of $<0.001$ (SMMSE, BADLS) and 0.081 (NPI) (Figure 1). A significant overall advantage was also seen for memantine addition versus placebo addition (with or without discontinuation of donepezil) for SMMSE $(p<0.001)$, BADLS $(p=0.015)$, and NPI $(p=0.002)$ (Figure 1 ). There was no evidence of negative or positive synergistic interaction between donepezil and memantine on the SMMSE ( $\mathrm{p}=0.14)$, NPI $(\mathrm{p}=0.42)$ and BADLS score $(p=0.09)$ across the entire trial period (12).

Visit by visit, there was a significant advantage of continued donepezil versus donepezil discontinuation on the SMMSE (Weeks 6, 18, 30, and 52; Figure 1A), the BADLS (Weeks 6, 18, 30, and 52; Figure 1B), and on the NPI (Week 18; Figure 1C) at a 2-sided $\alpha=0.05$. For memantine addition, there were visit-by-visit significant effects of adding memantine versus adding placebo on the SMMSE (Weeks 6, 18, and 30; Figure 1A), the NPI (Weeks 6, 30, and 52; Figure 1C), and on the BADLS (Weeks 18 and 30; Figure 1B), also at 2-sided $\alpha=0.05$.

\section{Memantine Clinical Trial Program}

Applying the statistical approach used in the DOMINO study to data from the Memantine Clinical Trial Program largely corroborated the DOMINO findings. Overall, there were statistically significant main effects for background donepezil versus placebo on the SIB $(p<0.001)$ ADCS-ADL19 $(p=0.020)$, NPI $(p<0.001)$, and the Composite Index $(\mathrm{p}=0.001)$ (Figure 2A, C-D), and a statistically significant advantage of memantine versus placebo in all four assessments (SIB, $\mathrm{p}<0.001$; 


\section{Figure 1: DOMINO Data, Reassessed}

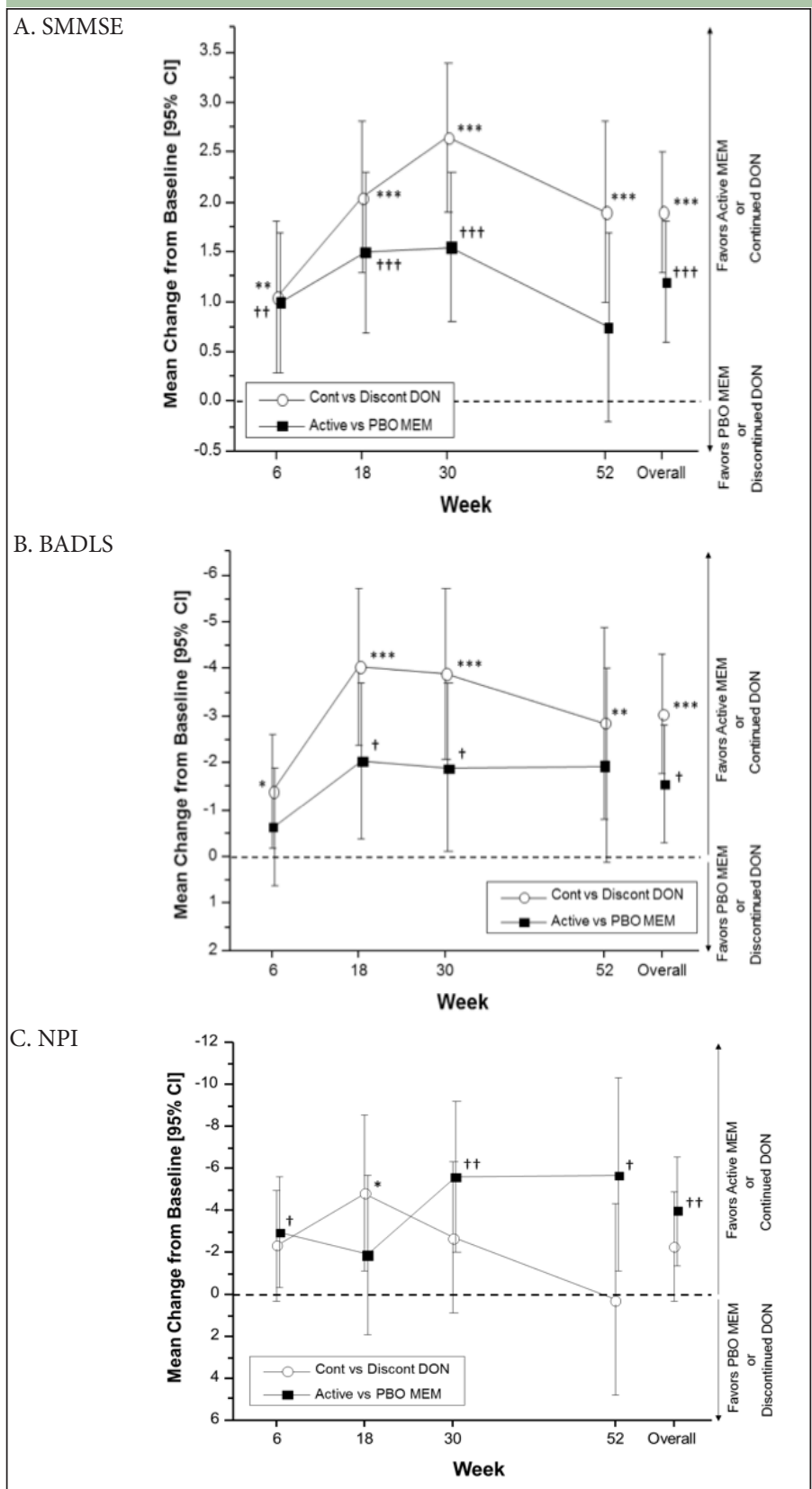

Continued DON vs Discontinued DON: ${ }^{*} \mathrm{p}<0.05 ;{ }^{* *} \mathrm{p}<0.01 ;{ }^{* * *} \mathrm{p}<0.001$; Active MEM vs PBO MEM: $t \mathrm{p}<0.05 ;++\mathrm{p}<0.01 ;++\dagger \mathrm{p}<0.001$; All differences are presented with $95 \%$ CIs. Data for Active MEM vs PBO MEM are average values across participants who continued or discontinued donepezil. Data for Continued DON and Discontinued DON are average values across participants who received active or placebo memantine; BADLS indicates Bristol Activities of Daily Living Scale (score range: 0-60); CI, confidence interval; DON, donepezil; MEM, memantine; NPI, Neuropsychiatric Inventory (score range: 0-144); PBO, placebo; SMMSE, Standardized Mini-Mental State Examination (score range: $0-30$ ).

ADCS-ADL19, $\mathrm{p}=0.017$; NPI, $\mathrm{p}<0.001$; Composite Index, $\mathrm{p}<0.001$ ) (Figure 2A-D). No significant positive or negative interactions were seen between background donepezil and memantine for any of the outcomes (all p-values $\geq 0.170$ ).

Visit-by-visit data indicated significant effects of donepezil versus placebo at Weeks 4,12 , and 24 on the SIB (Figure 2A), Week 24 on the ADCS-ADL19 (Figure 2B), and Weeks 12 and 24 on the NPI and the Composite
Index (Figure 2C \& 2D). There were significant effects of adding memantine versus adding placebo at Weeks 4, 12 , and 24 on the SIB and the Composite Index (Figure 2A \& 2D), Week 24 on the ADCS-ADL19 (Figure 2B), and Weeks 12 and 24 on the NPI (Figure 2C).

The covariance structure had minimal influence on the results. The outcomes of the unstructured covariance model are shown for all outcomes.

\section{Discussion}

The reassessment of the DOMINO results with common statistical tools and the meta analysis of the Memantine Clinical Trial Program data, in the context of standard statistical definitions of additivity and synergy, provide converging evidence that the addition of memantine to existing donepezil therapy is associated with benefits across multiple clinical domains. There was statistical evidence of an additive effect for the memantine and donepezil treatments in the DOMINO study and in the pooled population of the Memantine Clinical Trial Program, but no statistically significant evidence of a negative or positive synergistic interaction (i.e., heterogeneity). The current analyses suggest that the results of the DOMINO study are consistent with the previous, larger studies that investigated the effects of memantine-ChEI combination therapy in patients with moderate to severe AD (3-6, 8-11). The precise mechanisms by which two drugs as different as memantine and donepezil may interact in the human brain are not entirely understood (24). Cholinergic neurons receive glutamatergic inputs (24) and vice versa $(25,26)$. As acetylcholine and choline acetyltransferase levels do not begin to fall significantly until dementia is advanced, there would be a good theoretical basis to assume benefit from simultaneous administration of memantine and donepezil into the moderate and severe stages of the disease (27).

A primary aim of the DOMINO study (12) was to determine whether initiating memantine treatment at the moderate and severe stages of $\mathrm{AD}$ is beneficial. The conclusions of the original publication were that initiation of memantine provided significant benefit for both primary outcomes and for the NPI. In addition, statistically significant benefits were seen for the continuation of donepezil for both primary outcomes, but not for the NPI. The DOMINO authors also reported that they "did not find significant heterogeneity in the efficacy of donepezil or memantine in the presence or absence of the other drug," based on non-significant donepezil by memantine interaction effects. However, the conclusion that there were no significant benefits of the combination of donepezil and memantine treatment over donepezil treatment alone (12) was based on separating a homogeneous population into small subgroups for analysis rather than using the primary ITT population to obtain estimates from the 


\section{Figure 2: Pooled Memantine Clinical Trial Program Data}

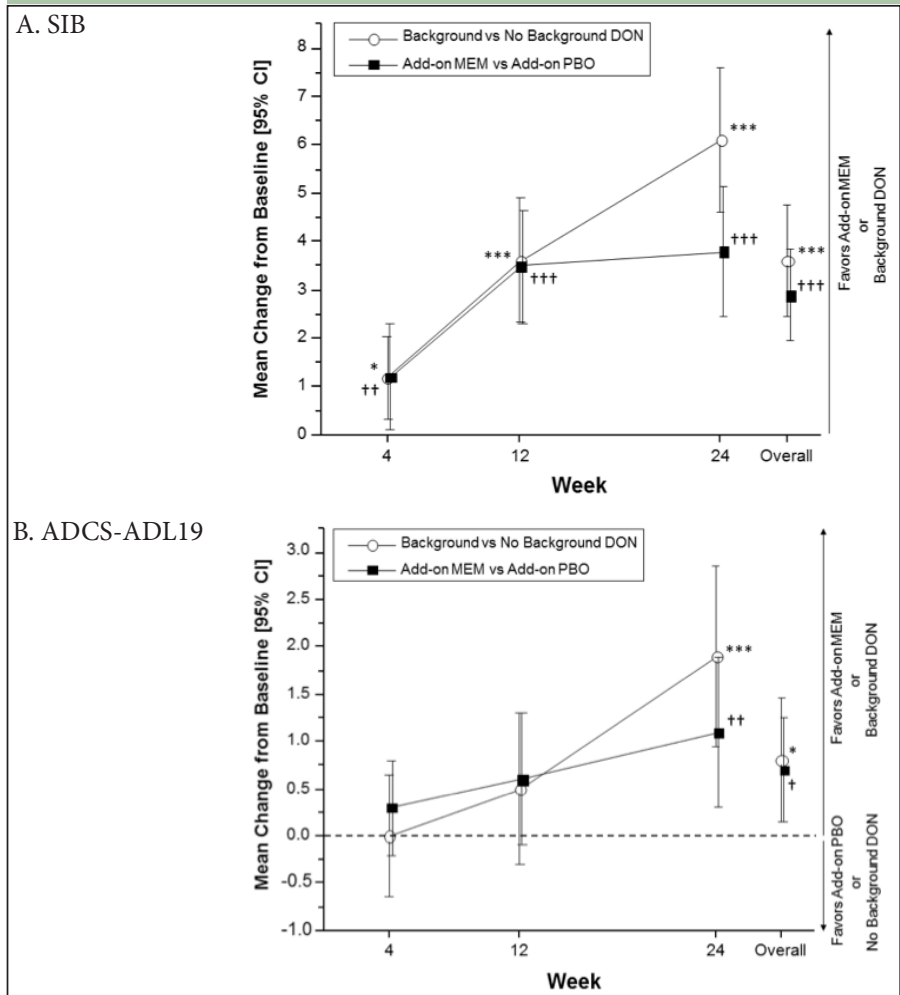

C. NPI

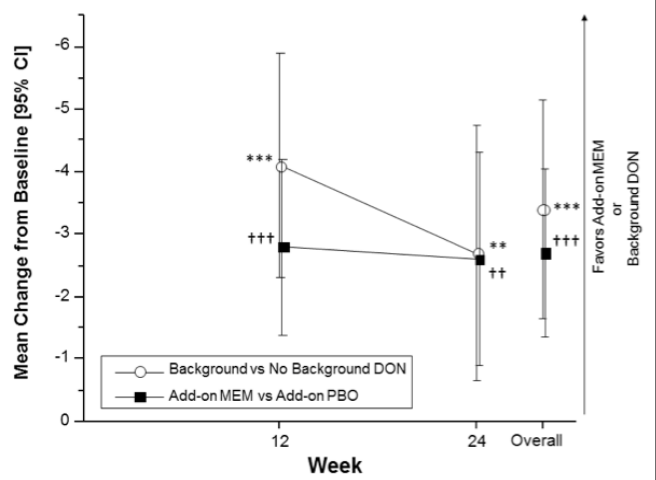

D. 4D Composite Index $\left.{ }^{35}\right]-{ }_{-}$Background vs No Background DON

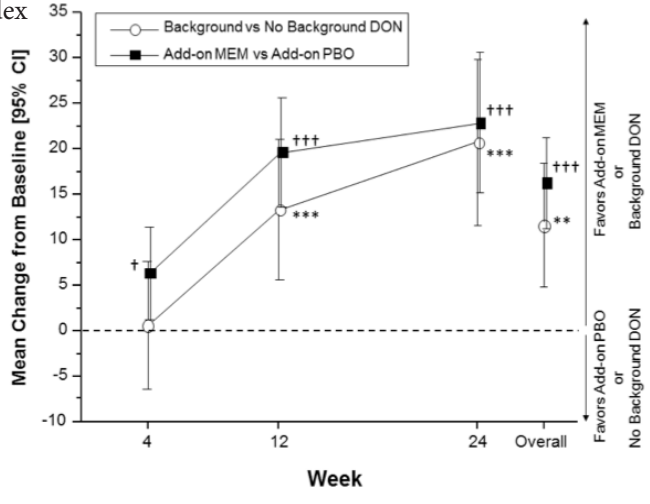

Background DON vs No Background DON: * $\mathrm{p}<0.05$; ${ }^{* *} \mathrm{p}<0.01$; ${ }^{* * *} \mathrm{p}<0.001$; Add-on MEM vs Add-on PBO: $\mathrm{t} p<0.05 ;+t p<0.01 ;+t+p<0.001$; All differences are presented with 95\% confidence intervals. Add-on MEM vs Add-on PBO values represent average values across participants with or without background DON treatment. Data for Background DON vs No Background DON represent average values across participants who received Add-on MEM or Add-on PBO; 4D, indicates four-dimensional; ADCS-ADL19, 19-item Alzheimer's Disease Cooperative Study - Activities of Daily Living scale (score range: 0-54); DON indicates donepezil; MEM, memantine, NPI, Neuropsychiatric Inventory (score range: 0-144); PBO, placebo; SIB, Severe Impairment Battery (score range: $0-100$ ). mixed model. This conclusion elicited criticism, in part because of the issues noted above as well as the small subgroup end-of-study sample sizes (20-38/group) and critical differences in dropout rates among the treatment groups (28). The current reassessment of the DOMINO study data suggested significant advantages of memantine compared to placebo in the presence or absence of donepezil for overall cognition (SMMSE), function (BADLS), and behavior (NPI) measures, thereby corroborating several lines of clinical evidence supporting the superiority of memantine-ChEI combination therapy over the component monotherapies (3, 6-11). Another aim of Howard et al (2012) was to address whether patients with moderate to severe $\mathrm{AD}$ already receiving donepezil benefit from continuing donepezil treatment (12). The superiority of continued donepezil treatment over discontinuation/placebo donepezil was demonstrated in the original publication (12), the current reassessment of the DOMINO study data, and in a soon to be published area-under-the-curve (AUC) analysis of the pooled data from the Memantine Clinical Trial Program, in which the 4D Composite Index for the 24/28 week treatment period showed additive and cumulative clinical advantages of memantine-donepezil combination over monotherapies with donepezil or memantine (7).

A key limitation of the current analysis is that the conclusions are based on post hoc assessments with nonrandomized groups for the donepezil comparisons, and baseline differences that may influence the donepezil comparisons. In addition, the statistical model used in DOMINO could not be used in its entirety for the pooled data since the non-donepezil patients did not have previous time on donepezil (12), which eliminated this covariate from the model.

In conclusion, this re-analysis suggests that the results of the DOMINO trial do not contradict previous studies in moderate to severe $\mathrm{AD}$ that found benefits of the combined memantine-donepezil treatment that exceed those of the monotherapies.

Conflict of interests: V. Otcheretko is an employee of the study sponsor. S. Hendrix, N. Ellison, and S. Stanworth are employees of Pentara Corporation, a contractor of the study's sponsor. P. N. Tariot received grants from the study sponsor.

Ethical standards: All participants, or their caregivers or legally authorized representatives, provided written informed consent before any study procedures were conducted, as noted in the original study publications $(3-6,12)$.

Funding: This analysis was sponsored by Forest Research Institute, LLC, an affiliate of Actavis, Inc. The sponsor was involved in the collection of a subset of data included in this analysis, in interpretation of results, preparation of the manuscript, and its review and approval.

Acknoledgments: Editorial support (development of tables and graphs, editorial suggestions, formatting for submission) was provided by Prescott Medical Communications Group (Chicago, IL, USA), a contractor of the study sponsor.

\section{References}

1. Gluud LL. Bias in clinical intervention research. American journal of epidemiology 2006;163, 493-501.

2. Ebrahim S, Sohani ZN, Montoya L, Agarwal A, Thorlund K, Mills EJ, 
Ioannidis JP. Reanalyses of randomized clinical trial data. JAMA 2014;312, 1024-1032.

3. Tariot PN, Farlow MR, Grossberg GT, Graham SM, McDonald S, Gergel I. Memantine treatment in patients with moderate to severe Alzheimer disease already receiving donepezil: a randomized controlled trial. JAMA 2004;291, 317-324.

4. Reisberg B, Doody R, Stoffler A, Schmitt F, Ferris S, Mobius HJ. Memantine in moderate-to-severe Alzheimer's disease. N Engl J Med 2003;348, 1333-1341.

5. van Dyck CH, Tariot PN, Meyers B, Malca Resnick E. A 24-week randomized, controlled trial of memantine in patients with moderate-to-severe Alzheimer disease. Alzheimer Dis Assoc Disord 2007;21, 136-143.

6. Grossberg GT, Manes F, Allegri RF, Gutierrez-Robledo LM, Gloger S, Xie L, Jia XD, Pejovic V, Miller ML, Perhach JL, Graham SM. The safety, tolerability, and efficacy of once-daily memantine $(28 \mathrm{mg})$ : a multinational, randomized, double-blind, placebo-controlled trial in patients with moderate-to-severe Alzheimer's disease taking cholinesterase inhibitors. CNS Drugs 2013;27, 469478.

7. Atri A, Hendrix S, Pejović V, Hofbauer RK, Edwards J, Molinuevo JL, Graham SM. Cumulative, additive benefits of memantine-donepezil combination over component monotherapies in moderate to severe Alzheimer's dementia: a pooled area under the curve analysis. Alzheimer Research \& Therapy Accepted, 2015.

8. Atri A, Molinuevo JL, Lemming O, Wirth Y, Pulte I, Wilkinson D. Memantine in patients with Alzheimer's disease receiving donepezil: new analyses of efficacy and safety for combination therapy. Alzheimers Res Ther 2013;5.

9. Atri A, Shaughnessy LW, Locascio JJ, Growdon JH. Long-term course and effectiveness of combination therapy in Alzheimer disease. Alzheimer Dis Assoc Disord 2008;22, 209-221.

10. Lopez OL, Becker J, Wahed A, Saxton J, Sweet R, Wolk D, Klunk W, Dekosky S. Long-term effects of the concomitant use of memantine with cholinesterase inhibition in Alzheimer disease. Journal of Neurology, Neurosurgery \& Psychiatry 2009;80, 600-607.

11. Rountree SD, Chan W, Pavlik VN, Darby EJ, Siddiqui S, Doody RS. Persistent treatment with cholinesterase inhibitors and/or memantine slows clinical progression of Alzheimer disease. Alzheimers Res Ther 2009;1, 7.

12. Howard R, McShane R, Lindesay J, Ritchie C, Baldwin A, Barber R, Burns A, Dening T, Findlay D, Holmes C, Hughes A, Jacoby R, Jones R, Jones R, McKeith I, Macharouthu A, O'Brien J, Passmore P, Sheehan B, Juszczak E, Katona C, Hills R, Knapp M, Ballard C, Brown R, Banerjee S, Onions C, Griffin M, Adams J, Gray R, Johnson T, Bentham P, Phillips P. Donepezil and memantine for moderate-to-severe Alzheimer's disease. N Engl J Med 2012;366, 893-903.

13. Birks J. Cholinesterase inhibitors for Alzheimer's disease Cochrane Database Syst Rev. 2006 25;(1):CD005593..

14. McShane R, Areosa Sastre A, Minakaran N. Memantine for dementia. Cochrane Database Syst Rev. 2006 2:CD003154.

15. Rabins P, Blacker D, Rovner B, Rummans T, Schneider L, Tariot P, Blass D,
McIntyre J, Charles S, Anzia D. APA Work Group on Alzheimer's Disease and other Dementias. American Psychiatric Association practice guideline for the treatment of patients with Alzheimer's disease and other dementias. Am J Psychiatry 2007;164, 5-56.

16. Geldmacher DS, Kerwin DR. Practical diagnosis and management of dementia due to Alzheimer's disease in the primary care setting: an evidencebased approach. The primary care companion for CNS disorders 2013;15:(4). pii: PCC.12r01474.

17. Molloy DW, Standish TI. A guide to the standardized Mini-Mental State Examination. Int Psychogeriatr 1997; 9 Suppl 1, 87-94; discussion 1997;143150.

18. Bucks RS, Ashworth DL, Wilcock GK, Siegfried K. Assessment of activities of daily living in dementia: development of the Bristol Activities of Daily Living Scale. Age Ageing 1996;25, 113-120.

19. Cummings JL, Mega M, Gray K, Rosenberg-Thompson S, Carusi DA Gornbein J. The Neuropsychiatric Inventory: comprehensive assessment of psychopathology in dementia. Neurology 1994;44, 2308-2314.

20. Saxton J, Swihart AA. Neuropsychological assessment of the severely impaired elderly patient. Clin Geriatr Med 1989;5, 531-543.

21. Galasko D, Bennett D, Sano M, Ernesto C, Thomas R, Grundman M, Ferris $\mathrm{S}$. An inventory to assess activities of daily living for clinical trials in Alzheimer's disease. The Alzheimer's Disease Cooperative Study. Alzheimer Dis Assoc Disord 1997;11 Suppl 2, S33-39.

22. Schneider LS, Olin JT, Doody RS, Clark CM, Morris JC, Reisberg B, Schmitt FA, Grundman M, Thomas RG, Ferris SH. Validity and reliability of the Alzheimer's Disease Cooperative Study-Clinical Global Impression of Change. The Alzheimer's Disease Cooperative Study. Alzheimer Dis Assoc Disord 1997;11 Suppl 2, S22-32.

23. Slinker BK. The statistics of synergism. Journal of molecular and cellular cardiology 1998;30, 723-731.

24. Parsons CG, Danysz W, Dekundy A, Pulte I. Memantine and cholinesterase inhibitors: complementary mechanisms in the treatment of Alzheimer's Disease. Neurotoxicity research 2013;24, 358-369.

25. Henny P, Jones BE. Projections from basal forebrain to prefrontal cortex comprise cholinergic, GABAergic and glutamatergic inputs to pyramidal cells or interneurons. European Journal of Neuroscience 2008;27, 654-670.

26. Arroyo S, Bennett C, Hestrin S. Nicotinic modulation of cortical circuits. Frontiers in neural circuits 2014;8:30. doi: 10.3389/ fncir.2014.00030.

27. Jones R, Sheehan B, Phillips P, Juszczak E, Adams I, Baldwin A, Ballard C, Banerjee S, Barber B, Bentham P. DOMINO-AD protocol: donepezil and memantine in moderate to severe Alzheimer's disease-a multicentre RCT. Trials 2009;10, 57

28. Tariot PN. Cessation of donepezil is associated with clinical decline in patients with moderate-to-severe Alzheimer's disease compared to continuation of donepezil or addition or substitution of memantine. Evidence Based Medicine 2013;18, 62-63. 